\title{
NONLINEAR FEEDBACK STABILIZATION OF A ROTATING BODY-BEAM WITHOUT DAMPING
}

\author{
Boumediène CHENTOUF ${ }^{1}$ And JEAn-François COUCHOURON ${ }^{1}$
}

\begin{abstract}
This paper deals with nonlinear feedback stabilization problem of a flexible beam clamped at a rigid body and free at the other end. We assume that there is no damping and the feedback law proposed here consists of a nonlinear control torque applied to the rigid body and either a boundary control moment or a nonlinear boundary control force or both of them applied to the free end of the beam. This nonlinear feedback, which insures the exponential decay of the beam vibrations, extends the linear case studied by Laousy et al. to a more general class of controls.

Résumé. On aborde dans cet article un problème de stabilisation par feedback non linéaire d'un système constitué d'une poutre flexible fixée à l'une de ses extrémités à un corps rigide et libre à l'autre extrémité. On suppose qu'il n'y a pas de frottements; on propose alors une loi de commande composée d'une part d'un contrôle de couple exercé sur le disque et d'autre part d'un contrôle de force et/ou d'un contrôle de moment appliqué à l'extrémité libre de la poutre. On montre ici que cette loi de commande non linéaire assure l'amortissement exponentiel des vibrations de la poutre. Cette conclusion est une extension des résultats antérieurs obtenus avec des contrôles linéaires dans le sens où notre classe de contrôle est plus large.
\end{abstract}

AMS Subject Classification. 35A05, 35B40, 47H06, 47H20, 93C20, 93D15, 93D05.

Received October 27, 1998. Revised June 14, 1999.

\section{INTRODUCTION}

The purpose of this paper is to study the nonlinear feedback stabilization problem of the system presented in the Figure 1. This system has been introduced by Baillieul and Levi [2]. It consists of a disk (D) with a beam (B) attached to its center and perpendicular to the disk plane. The disk (D) rotates freely around its axis and the motion of the beam (B) is confined to a plane perpendicular to the disk (see Fig. 1).

\footnotetext{
Keywords and phrases: Rotating body-beam, non linear control, exponential stability.

1 INRIA-Lorraine (CONGE project) \& UPRES A 7035, ISGMP, bâtiment A, Université de Metz, Ile de Saulcy,

57045 Metz cedex 01, France; e-mail: chentouf, couchour@loria.fr
} 


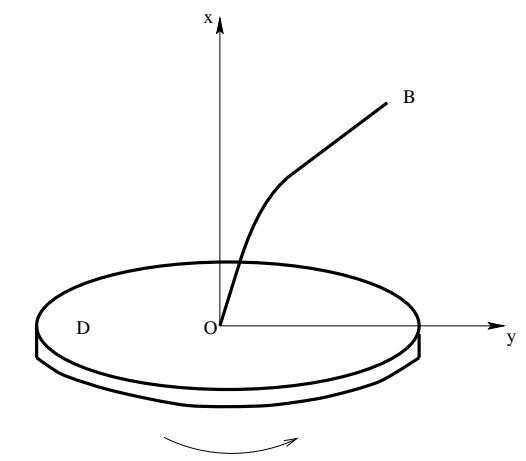

Figure 1. The body-beam system.

The body-beam system is governed by the following equations

$$
\left\{\begin{array}{l}
\rho y_{t t}+E I y_{x x x x}=\rho \omega^{2}(t) y \\
y(0, t)=y_{x}(0, t)=0 \\
y_{x x}(1, t)=\Gamma_{1}(t) \\
y_{x x x}(1, t)=\Gamma_{2}(t) \\
\dot{\omega}(t)=\frac{\Gamma_{3}(t)-2 \rho \omega(t) \int_{0}^{1} y y_{t} d x}{I_{d}+\rho \int_{0}^{1} y^{2} d x}
\end{array}\right.
$$

where the positive constants $E I, \rho$ and $I_{d}$ are respectively the flexural rigidity, the mass per unit length of the beam, and the disk's moment of inertia; $\omega(t)$ is the angular velocity of the disk at time $t$, while $y(., t)$ is the beam's displacement in the rotating plane at time $t$. For simplicity, the length of the beam is chosen to be unity. Moreover, $\Gamma_{1}(t), \Gamma_{2}(t)$ and $\Gamma_{3}(t)$ are respectively the moment control, the force control and the torque control to be determined so that the solution's energy of the resulting closed-loop system decays to zero in some functional space.

In this paper, we take a nonlinear boundary control moment $\Gamma_{1}(t)$ and/or a nonlinear boundary control force $\Gamma_{2}(t)$ applied to the free end of the beam (B) while a nonlinear control torque $\Gamma_{3}(t)$ is exerted on the disk (D). Then, as in the linear case [16] it will be shown that, in our case, the equilibrium point of (1.1) is still exponentially stabilizable.

The stabilization problem of the body-beam system has been extensively studied in the literature $[2,5,9$, 16-19, 23, 24]. In [2], the authors showed that with structural damping and without control, the body-beam system has a finite number of rotating equilibrium states. Later, Bloch and Titi [5] showed that in the more difficult case of viscous damping, a linear inertial manifold exists for the body-beam system. By taking into account the effect of damping, and for any constant angular velocity smaller than a critical one, an exponentially stabilizing feedback torque control law has been given in [23]. In the same case, and by adding a boundary control force, the system is also stabilizable for any constant angular velocity [24]. The stabilization problem of similar systems has been studied in [17,18] and [19]. Recently, for the body-beam system without damping, exponential stabilization was established in [16] as soon as at least one of two linear boundary controls (force or moment) is present at the free end of the beam with, in addition, a control torque of the disk. The last result on this subject has been obtained by Coron and d'Andréa-Novel [9]: without damping nor controls on the free boundary of the beam, the authors found a torque control which insured the strong stability of the system but not the exponential one.

The contribution of this paper consists in extending the class of controls proposed in [16] for the system without damping to a nonlinear one. In fact, this work is motivated by two arguments: first, the interest of such an extension is to highlight the robustness of controls which is a very desirable property. This means nothing 
more than that the stability of the system will stand even in the presence of small perturbations. In any realistic system, these perturbations could result from modeling errors, or moderate disturbances and uncertainties in the model which is often far from perfect. In addition, we propose a nonlinear large class of controls so that the errors of measurement, concerning the implementation of sensors or actuators, will be taken into account. Second, it is important that, when we search a control law for our model, we keep in mind that in practice the input amplitudes are constrained by the power of the actuators which go into a nonlinear saturation [1]. Therefore, the stability of the system must be assured with nonlinear controls.

Thus, the new feedback control proposed here is the following:

$$
\left\{\begin{array}{l}
\Gamma_{1}(t)=-f\left(y_{t x}(1, t)\right), \quad \Gamma_{2}(t)=g\left(y_{t}(1, t)\right) \\
\Gamma_{3}(t)=-\gamma\left(\omega-\omega^{*}\right)
\end{array}\right.
$$

for each given $\omega^{*} \in \mathbb{R}$. In contrast to [16], these controls are nonlinear since $f, g$ and $\gamma$ are nonlinear real functions satisfying hypotheses F1), F2) and F3) given in Section 3.

The main result of our paper is then (Th. 2 in Sect. 3):

under hypotheses F1), F2), F3) and the condition $\left|\omega^{*}\right|<\sqrt{9 E I / \rho}$, the solutions of the closed-loop system (1.1)-(1.2) decay exponentially to its equilibrium point in a suitable state space.

We forewarn the reader that as in [16], the decay rate although exponential is not uniform. To obtain this result within a nonlinear framework, it was necessary to overcome new difficulties among which, well-posedness of the system (1.1-1.2), regularity of its solutions, density of the domain of the nonlinear unbounded operator governing the evolution problem, lack of exponential stability result in the perturbation theory of fully nonlinear systems, no Duhamel's formula... For instance, the linear argument of [16] to deduce the exponential decay of the solutions of (1.1-1.2) from the exponential stability of the associated homogenous system fails in our approach. In fact, Theorem 2 has been obtained by means of two essential tools: on the one hand, the Bénilan's integral inequalities (see [3] or [4]) and on the other hand, the multiplier method (see [15]). form:

All the systems considered in this paper (and in particular (1.1-1.2)) can be written in the following abstract

$$
C P=\left\{\begin{array}{l}
\dot{x}(t)+A x(t)+B(t, x(t)) \ni 0, \\
x(0)=x^{0}
\end{array}\right.
$$

where $J$ is any interval in $\mathbb{R}^{+}$starting at zero; $A$ is a (multivalued) nonlinear m-accretive unbounded operator on the Banach space $(X,\|\|)$; and $x^{0}$ belongs to the closure of the domain $\mathcal{D}(A)$ of $A$. The nonlinear non accretive operator $B$ is in general (except in Sec. 2.3) locally Lipschitz from $J \times X$ to $X$. Note that the multivalued aspect of $A$ (which is a usual requirement in the modern theory of evolution equation) does not pose any problem in the study of differential inclusions (1.3). Indeed, the mild solution notion involves the operator resolvent $(I+\lambda A)^{-1}(\lambda>0)$ which is single-valued and non expansive provided that $A$ is accretive. We point out that in the body-beam application, $A$ is single-valued, $X$ is a Hilbert space and $\mathcal{D}(A)$ is dense in $X$. Moreover, the nonlinearity of $A$ unfortunately occurs in its domain and the density of $\mathcal{D}(A)$ has been obtained by non standard arguments (see Lem. 4).

Section 2 is devoted to preliminary results needed to prove global existence, regularity and exponential decay of the solutions of problem $C P$. In Section 3, we state and show in several steps the exponential decay result of the rotating body-beam system (1.1-1.2). 


\section{Preliminaries on the abstract problem $\boldsymbol{C} \boldsymbol{P}$}

\subsection{The Cauchy problem $C P$}

First, we start by briefly recalling some definitions and notations that are consistent throughout this paper. Let $X$ be a real Banach space. By a multivalued operator, we mean a mapping $A: X \rightarrow 2^{X}$. The domain of $A$ is defined by

$$
\mathcal{D}(A):=\{x \in X ; A x \neq \emptyset\},
$$

and the range of $A$ is defined by

$$
R(A):=\bigcup_{x \in \mathcal{D}(A)} A x
$$

Definition 1. An operator $A: X \rightarrow 2^{X}$ is called accretive if $[x-\hat{x}, y-\hat{y}] \geq 0$, for each $x, \hat{x} \in \mathcal{D}(A), y \in A x$, $\hat{y} \in A \hat{x}$, where $[\cdot, \cdot]$ is the usual bracket

$$
[u, v]=\lim _{\lambda \downarrow 0} \frac{\|u+\lambda v\|-\|u\|}{\lambda}, \forall u, v \in X
$$

Definition 2. An operator $A: X \rightarrow 2^{X}$ is called $\underline{\text { m-accretive }}$ if it is accretive and $R(I+\lambda A)=X$.

Then, consider the nonlinear differential inclusion (1.3). This terminology can be used to highlight the fact that $A$ is multivalued and so we use the symbol " $\ni 0$ " instead of " $=0$ ". We point out that the proofs are not simpler if $A$ is a function. If necessary, we will precise some arguments in $(C P)$ as follows: $C P=C P\left(x^{0}, J\right)$. Throughout this subsection, the system (1.3) is assumed to satisfy the following hypotheses.

(HI) $A$ is a multivalued unbounded nonlinear m-accretive operator with domain $\mathcal{D}(A)$.

(HII) $B$ is locally Lipschitz on $J \times X$ and bounded on the bounded subsets of $J \times X$.

(HIII) For every $x^{0} \in \overline{\mathcal{D}(A)}$ and $T \in J$, there is a constant $C=C\left(x^{0}, T\right)$ such that for all $T_{0} \in[0, T]$, each solution $x(\cdot)$ of $C P\left(x^{0},\left[0, T_{0}\right]\right)$ satisfies:

$$
\|x(t)\| \leq C, \forall t \in\left[0, T_{0}\right] .
$$

We will precise below the meaning of solutions considered in this paper.

Definition 3. The continuous function $x_{*}$ is a solution of $C P$ if it is the mild solution of the quasi-autonomous problem

for all $T \in J$.

$$
Q=\left\{\begin{array}{l}
\dot{x}(t)+A x(t) \ni-B\left(t, x_{*}(t)\right), \quad t \in[0, T], \\
x(0)=x^{0}
\end{array}\right.
$$

We refer, for instance, the reader to [10] for the notion of mild solution for the quasi-autonomous case.

Proposition 1 below extends the well-known Picard-Lindelöf-Lipschitz theorem.

Proposition 1. Assume that (HI), (HII) and (HIII) hold. Then, for $x^{0} \in \overline{\mathcal{D}(A)}$, the problem $C P\left(x^{0}, J\right)$ has a unique solution $S(\cdot) x^{0}$ and the map $x^{0} \mapsto S(t) x^{0}$ is continuous on $\overline{\mathcal{D}(A)}$, for all $t \in J$.

Proof of Proposition 1. The proof will be divided into three steps and without loss of generality, we will suppose $J=[0, T]$ for some $T>0$.

Step 1. Existence and uniqueness of solution $S(\cdot) x^{0}$ are not really a new result since due to assumptions (HI) and (HII), the problem $C P$ has a unique local solution $x(\cdot)$, i.e., there are $\left.T_{0} \in\right] 0, T[$ and $x(\cdot)$ solution of $C P\left(x^{0},\left[0, T_{0}[)\right.\right.$ (see Pierre result, p. 126 of [21]). Moreover, in order to show that this solution can be extended 
on $J$ it is enough to fit classical arguments (as in [21] for instance) to our particular assumptions (HII) and (HIII). Indeed, the existence of $\lim _{t \rightarrow T_{0}^{-}} x(t)$ follows from the following estimates:

$$
\|x(t+h)-x(t)\| \leq\|x(s+h)-x(s)\|+2 M(t-s),
$$

with $M=\sup \{\|B(t, \xi)\| ; t \in[0, T],\|\xi\| \leq C\}$ and $C$ is given in (HII). According to our assumptions, such an estimate is provided by Bénilan's integral inequalities (see [3] and [4]).

Step 2. Now it remains to prove the continuity of $x^{0} \mapsto S(t) x^{0}$ on $\overline{\mathcal{D}(A)}$, for all $t \in J$. This is not obvious since we do not suppose any compacity property on $B$ or anything upon the dependence $x^{0} \mapsto C\left(x^{0}, T\right)$. First, we will prove the following uniform estimate which does not require assumption (HIII).

The notation $\mathcal{B}(z, r)$ stands for the open ball of center $z$ and radius $r>0$ in $X$.

Lemma 1. Let $r>0$ and $x^{0} \in \overline{\mathcal{D}(A)}$ be such that $B$ is L-Lipschitz on $[0, r] \times \mathcal{B}\left(x^{0}, r\right)$. Let $x^{1} \in \mathcal{B}\left(x^{0}, r / 8\right) \cap$ $\mathcal{D}(A) ;$ pick $\widehat{x^{1}} \in A x^{1}$ and a real $\left.T_{1} \in\right] 0, r[$ satisfying

$$
\left(\frac{r}{4}+T_{1}\left(\left\|\widehat{x^{1}}\right\|+M_{1}\right)\right) e^{L T_{1}}<\frac{r}{2},
$$

with

$$
M_{1}=\sup \left\{\left\|B\left(\tau, x^{1}\right)\right\| \cdot \tau \in[0, r]\right\} .
$$

Then we have $S(t) x_{0} \in \mathcal{B}\left(x^{0}, r\right)$ for all $t \in\left[0, T_{1}\right]$ and all $x_{0} \in \mathcal{B}\left(x^{0}, r / 8\right)$.

Proof of Lemma 1. Let $x_{0} \in \mathcal{B}\left(x^{0}, r / 8\right)$ and set $y(\cdot)=S(\cdot) x_{0}$. Define the set

$$
\mathcal{E}=\left\{t \in\left[0, T_{1}\right] ;\left\|y(\tau)-x_{0}\right\|<r ; \text { for all } \tau \in[0, t]\right\} .
$$

Clearly, $\mathcal{E}$ is a non empty open subset of $\left[0, T_{1}\right]$. Let us prove that it is closed in order to conclude $\mathcal{E}=\left[0, T_{1}\right]$. To accomplish this aim, set

$$
t_{\infty}=\sup \mathcal{E}
$$

Then, for $t \in\left[0, t_{\infty}[\right.$, Bénilan's integral inequalities and usual bracket properties (see Def. 1 and $[4,6]$ ) yield

$$
\begin{aligned}
\left\|y(t)-x^{1}\right\| \leq & \left\|x_{0}-x^{1}\right\|+\int_{0}^{t}\left[y(\tau)-x^{1},-B(\tau, y(\tau))-\widehat{x^{1}}\right] d \tau \\
\leq & \frac{r}{4}+\int_{0}^{t}\left(\left[y(\tau)-x^{1},-B(\tau, y(\tau))+B\left(\tau, x^{1}\right)\right]\right. \\
& \left.+\left[y(\tau)-x^{1},-B\left(\tau, x^{1}\right)-\widehat{x^{1}}\right]\right) d \tau \\
\leq & \frac{r}{4}+\int_{0}^{t}\left(L\left\|y(\tau)-x^{1}\right\|+\left\|B\left(\tau, x^{1}\right)\right\|+\left\|\widehat{x^{1}}\right\|\right) d \tau .
\end{aligned}
$$

By virtue of Gronwall's lemma, the latter implies

$$
\left\|y(t)-x^{1}\right\| \leq\left(\frac{r}{4}+t\left(\left\|\widehat{x^{1}}\right\|+M_{1}\right)\right) e^{L t}, \forall t \in\left[0, t_{\infty}[.\right.
$$

According to the choice of $T_{1}$ in $(2.1)$ and the relation $0 \leq t_{\infty} \leq T_{1}$, it comes, since $x$ is continuous on $[0, T]$

$$
\left\|y\left(t_{\infty}\right)-x^{1}\right\| \leq \frac{r}{2}
$$

Now, using the triangle inequality in (2.2), we conclude

$$
\left\|y\left(t_{\infty}\right)-x^{0}\right\| \leq \frac{r}{2}+\left\|x^{0}-x^{1}\right\| \leq \frac{r}{2}+\frac{r}{8}<r .
$$


From (2.3) and the definitions of $\mathcal{E}$ and $t_{\infty}$, it results $t_{\infty} \in \mathcal{E}$ and thus $\mathcal{E}$ is closed.

Step 3. Let $\left(x_{n}^{0}\right)_{n \in \mathbb{N}^{*}}$ be a sequence converging in $\overline{\mathcal{D}(A)}$ to $x^{0}$. Define $x_{n}$ and $x$ as

$$
x_{n}(t)=S(t) x_{n}^{0} ; x(t)=S(t) x^{0}, \quad t \in[0, T] .
$$

In order to prove the continuity of $S(t)$, we will show that the sequence $\left(x_{n}\right)_{n}$ converges uniformly to $x$ on $[0, T]$. It suffices then to establish that the set

$$
\left.\mathcal{K}=\{\theta \in] 0, T] ; x_{n} \rightarrow x \text { uniformly on }[0, \theta]\right\}
$$

is non empty, open and closed in $[0, T]$.

i) $\mathcal{K}$ is non empty:

The positive real $T_{1}$ introduced in Lemma 1 belongs to $\mathcal{K}$. Indeed, using again Bénilan's integral inequalities, we have

$$
\left\|x_{n}(t)-x(t)\right\| \leq\left\|x_{n}^{0}-x^{0}\right\|+\int_{0}^{t}\left\|B\left(\tau, x_{n}(\tau)\right)-B(\tau, x(\tau))\right\| d \tau
$$

for $t \in\left[0, T_{1}\right]$. But Lemma 1 insures

$$
x_{n}(t) \in \mathcal{B}\left(x^{0}, r\right), \text { for } t \in\left[0, T_{1}\right] \text { and } n \geq m,
$$

when the integer $m$ is sufficiently large, if $B$ is L-Lipschitz on $[0, r] \times \mathcal{B}\left(x^{0}, r\right)$. It follows from $(2.4,2.5)$ and the Gronwall's lemma

$$
\left\|x_{n}(t)-x(t)\right\| \leq\left\|x_{n}^{0}-x^{0}\right\| e^{L t}
$$

for $t \in\left[0, T_{1}\right]$ and $n \geq m$. Hence $\left(x_{n}\right)_{n}$ converges uniformly to $x$ on $\left[0, T_{1}\right]$.

ii) $\mathcal{K}$ is open:

Let $\theta_{1} \in \mathcal{K}$ be such that $\theta_{1}<T$. Then, in particular, $x_{n}\left(\theta_{1}\right) \rightarrow x\left(\theta_{1}\right)$. As in i), we can show that there exists $T_{1}^{\prime} \in\left[0, T-\theta_{1}\left[\right.\right.$ such that $\left(x_{n}\right)$ converges uniformly to $x$ on $\left[\theta_{1}, \theta_{1}+T_{1}^{\prime}\right]$. Therefore, one has $\left[0, \theta_{1}+T_{1}^{\prime}\right] \subset \mathcal{E}$ which proves the claim ii).

iii) $\mathcal{K}$ is closed:

Let $\theta_{n} \in \mathcal{E}, n \in \mathbb{N}^{*}$ be such that $\theta_{n} \uparrow \theta_{\infty}$. We have to prove that $\left(x_{n}\right)_{n}$ converges uniformly to $x$ on $\left[0, \theta_{\infty}\right]$. First, we can find $r>0$ then $\left.T_{2} \in\right] 0, r\left[\right.$ and then $m \in \mathbb{N}^{*}$ as follows:

a) $B$ is L-Lipschitz on $\left[\theta_{\infty}-r, \theta_{\infty}\right] \times \mathcal{B}\left(x\left(\theta_{\infty}\right), r\right)$;

b) $\left\|x\left(\theta_{\infty}-\tau\right)-x\left(\theta_{\infty}\right)\right\| \leq \frac{r}{16}$, for all $\tau$ in $\left[0, T_{2}\right]$;

c) $\left(\frac{r}{4}+T_{2}\left(\left\|\widehat{x^{1}}\right\|+M_{2}\right)\right) e^{L T_{2}}<\frac{r}{2}$, with $x^{1} \in \mathcal{B}\left(x\left(\theta_{\infty}\right), r / 8\right) \cap \mathcal{D}(A)$ and $M_{2}=\sup _{\tau \in[0, r]}\left\|B\left(\theta_{\infty}-\tau, x^{1}\right)\right\|$

d) $\left\|x_{n}\left(\theta_{\infty}-T_{2}\right)-x\left(\theta_{\infty}-T_{2}\right)\right\| \leq \frac{r}{16}$ for $n \geq m$.

Then, as in Lemma 1, one can check

$$
x_{n}\left((t) \in \mathcal{B}\left(x\left(\theta_{\infty}\right), r\right),\right.
$$

for $n \geq m$ and $t \in\left[\theta_{\infty}-T_{2}, \theta_{\infty}[\right.$.

But the Bénilan's inequalities provide

$$
\left\|x_{n}(t)-x(t)\right\| \leq\left\|x_{n}\left(\theta_{\infty}-T_{2}\right)-x\left(\theta_{\infty}-T_{2}\right)\right\|+\int_{\theta_{\infty}-T_{2}}^{\theta_{\infty}}\left\|B\left(\tau, x_{n}(\tau)\right)-B(\tau, x(\tau))\right\| d \tau,
$$

for $t \in\left[\theta_{\infty}-T_{2}, \theta_{\infty}\right]$. 
According to (2.6), the Lebesgue Dominated Convergence Theorem used in the last inequality gives the uniform convergence of $\left(x_{n}\right)_{n}$ to $x$ on $\left[\theta_{\infty}-T_{2}, \theta_{\infty}\right]$ and finally on $\left[0, \theta_{\infty}\right]$. Thus, as required at the begining of iii) we have $\theta_{\infty} \in \mathcal{K}$. The proof of Proposition 1 is now complete.

\subsection{Two lemmas without (HIII)}

The two following technical lemmas which do not involve hypotheses (HIII) are used in Section 3 in order to check (HIII) for $C P$ problem stemmed from the body-beam system.

Lemma 2. Let $V: X \rightarrow \mathbb{R}$ be a continuous function, $x^{0} \in \overline{\mathcal{D}(A)}$ and $x$ be the solution of $C P\left(x^{0},[0, T]\right)$. Assume that $(\mathbf{H I})$ and $(\mathbf{H I I})$ hold. Suppose in addition that each local solution y of $C P\left(x_{0},[0, T]\right)$ with $x_{0} \in \mathcal{D}(A)$ satisfies:

$$
V(y(t)) \leq V\left(x_{0}\right)
$$

on the domain of $y$. Then, we have

$$
V(x(t)) \leq V\left(x^{0}\right)
$$

for all $t \in[0, T]$. Consequently, Hypothesis (HIII) holds if, for instance, $V(z) \geq K\|z\|$ for any $z \in X$ and for some positive constant $K$.

Proof of Lemma 2. The function $x$ being the solution of $C P\left(x^{0},[0, T]\right)$, set

$$
\mathcal{G}=\left\{t \in[0, T] ; V(x(\tau)) \leq V\left(x^{0}\right), \forall \tau \in[0, t]\right\}
$$

Let us show $\mathcal{G}=[0, T]$. Clearly, $\mathcal{G}$ is closed in $[0, T]$. Now, we want to prove that $\mathcal{G}$ is not reduced to $\{0\}$. Let $x_{n}^{0} \rightarrow x^{0}$ with $x_{n}^{0} \in \mathcal{D}(A)$ for all $n \in \mathbb{N}^{*}$. From Lemma 1 (which does not suppose (HIII)), there exists $\left.\left.T_{1} \in\right] 0, T\right]$ such that the solution $S(\cdot) x_{n}^{0}$ exists on $\left[0, T_{1}\right]$ for $n$ sufficiently large and such that (see also Step 3 i) in the proof of Prop. 1) $\left(S(\cdot) x_{n}^{0}\right)_{n}$ converges to $x(\cdot)=S(\cdot) x^{0}$ uniformly on $\left[0, T_{1}\right]$. Therefore, we have

$$
V\left(S(t) x_{n}^{0}\right) \leq V\left(x_{n}^{0}\right), \text { for } t \in\left[0, T_{1}\right], n \in \mathbb{N}^{*} .
$$

Since $V$ is continuous, the previous relation yields

$$
V\left(S(t) x^{0}\right) \leq V\left(x^{0}\right), \text { for } t \in\left[0, T_{1}\right]
$$

and thus $\left[0, T_{1}\right] \subset \mathcal{G}$. Finally, repeating these arguments, one can easily show that $\mathcal{G}$ is open in $[0, T]$. Therefore, $\mathcal{G}=[0, T]$ and thus the proof of Lemma 2 is ended.

In a Hilbert space and more generally in a Banach space $X$ which has the Radon-Nykodym property, each Lipschitz continuous map from $[0, T]$ to $X$ is a.e. differentiable on $[0, T]$.

Lemma 3. Suppose that (HI) and (HII) hold and that the Banach space $X$ has the Radon-Nykodym property. Then, each solution $x$ of $C P\left(x^{0},[0, T]\right)$, with $x^{0} \in \mathcal{D}(A)$, is a strong one.

Proof of Lemma 3. Let $x^{0} \in \mathcal{D}(A), r>0$ and $T_{1}$ chosen as in Lemma 1 with $x^{1}=x^{0}$ for instance. For $t, t+h \in\left[0, T_{1}\right]$ with $h \geq 0$, the Bénilan's inequalities imply:

$$
\begin{aligned}
\|x(t+h)-x(t)\| & \leq\left\|x(h)-x^{0}\right\|+\int_{0}^{t}(\|B(\tau+h, x(\tau+h))-B(\tau, x(\tau))\|) d \tau \\
& \leq\left\|x(h)-x^{0}\right\|+\int_{0}^{t}(L h+L\|x(\tau+h)-x(\tau)\|) d \tau .
\end{aligned}
$$


But we have (see [3] or [4])

$$
\left\{\begin{array}{l}
\left\|x(h)-x^{0}\right\| \leq h\left\|\widehat{x^{1}}\right\|+M_{1} h \\
\widehat{x^{1}} \in A x^{0} \text { and } M_{1}=\sup _{\tau \in[0, T]}\|B(\tau, x(\tau))\|
\end{array}\right.
$$

Clearly (2.8) and the Gronwall's lemma applied in (2.7) show that $x$ is Lipschitz on $[0, T]$. Because $X$ has the Radon-Nykodym property, the map $x$ is differentiable a.e. on $\left[0, T_{1}\right]$. Then, in this case (see [3] or [4]), $x$ belongs to $W^{1,1}\left(\left[0, T_{1}\right] ; X\right)$ and satisfies

$$
\left\{\begin{array}{l}
x(t) \in \mathcal{D}(A), \text { a.e on }\left[0, T_{1}\right] \\
\dot{x}(t)+A x(t)+B(t, x(t)) \ni 0
\end{array}\right.
$$

In other words, $x$ is a strong solution of $C P\left(x^{0},\left[0, T_{1}\right]\right)$.

Now introduce the set

$$
\mathcal{L}=\left\{t \in[0, T] ;\left.x\right|_{[0, t]} \text { is a strong solution of } C P\left(x^{0},[0, t]\right)\right\}
$$

This set is clearly closed and contains $\left[0, T_{1}\right]$. In order to conclude $\mathcal{L}=[0, T]$, it remains to prove that $\mathcal{L}$ is open.

Consider $t_{0} \in \mathcal{L}$ with $t_{0}<T$ and some open ball $\mathcal{B}\left(x\left(t_{0}\right), r\right)$ such that the operator $B$ is $L$-Lipschitz on $\left[t_{0}-r, t_{0}+r\right] \times \overline{\mathcal{B}\left(x\left(t_{0}\right), r\right)}$. Then, from $(2.9)$ and the continuity of $x$, there is $\left.\left.h \in\right] 0, t_{0}\right]$ such that $x\left(t_{0}-h\right) \in \mathcal{D}(A)$ and $x(\tau) \in \mathcal{B}\left(x\left(t_{0}\right), r\right)$ for $\tau \in\left[t_{0}-h, t_{0}\right]$. But by virtue of part a) of this proof, $x$ is a strong solution of $C P\left(x\left(t_{0}-h\right),\left[0, T_{1}^{\prime}\right]\right)$ where $T_{1}^{\prime}$ is any real in $\left.\left.] t_{0}, T\right] \cap\right] t_{0}, t_{0}+r[$ satisfying

$$
t_{0}-h \leq t \leq T_{1}^{\prime} \Rightarrow x(t) \in \mathcal{B}\left(x\left(t_{0}\right), r\right)
$$

Of course, due to the continuity of $x$ such a $T_{1}^{\prime}$ exists and thus we have $\left[0, T_{1}^{\prime}\left[\subset \mathcal{L}\right.\right.$ and $t_{0} \in\left[0, T_{1}^{\prime}[\right.$. The proof is now done.

\subsection{An exponential decay result}

In this subsection, our hypotheses are different from those of the previous developments. Let $x^{0} \in X$ and denote by (HIV) and (HV) the following hypotheses:

(HIV) The problem $C P\left(x^{0},[0,+\infty[)\right.$ has a unique solution $x$.

(HV) There is a locally integrable positive real valued function $\mu$ on $[0,+\infty[$ satisfying

$$
\|B(t, x(t))\| \leq \mu(t)\|x(t)\|, \text { and } \lim _{\xi \rightarrow+\infty} \int_{\xi}^{\xi+\delta} \mu(\tau) d \tau=0, \forall \delta>0
$$

The result of this subsection is the following.

Theorem 1. Assume that the nonlinear semigroup $e^{-A t}$ generated by the operator $-A$ is exponentially (uniformly) stable on $X$. Then, under hypotheses (HI), (HIV) and (HV), there exist positive constants $M$ and $\kappa$ such that

$$
\|x(t)\| \leq M e^{-\kappa t}, \forall t \geq 0
$$


Remark 1. The decay rate obtained in Theorem 1, although exponential, is not uniform. In return, the nonlinear semigroup $e^{-A t}$ being uniformly stable, there exists constants $M_{1}, \omega_{1}>0$ satisfying $\left\|e^{-A t} x^{0}\right\| \leq$ $M_{1} e^{-\omega_{1} t}\left\|x^{0}\right\|$, for all $x^{0} \in X$ and $t \geq 0$. Then, the constants $M$ and $\kappa$ of Theorem 1 can be chosen as follows

$$
\kappa=\frac{\omega_{0}}{\delta}, \quad M=\max \left(M_{1} e^{\omega_{0}\left(2+t_{0} / \delta\right)}, \sup _{0 \leq t \leq t_{0}}\|x(t)\| e^{\kappa t_{0}}\right)
$$

with

and $t_{0}$ defined by

$$
\omega_{0}>\max \left(1, \omega_{1}\right), \delta>\frac{1}{\omega_{1}}\left(\ln M_{1}+2 \omega_{0}\right)
$$

$$
\int_{\xi}^{\xi+\delta} \mu(\tau) d \tau \leq \frac{\omega_{0}}{e^{\omega_{0} \delta}}, \forall \xi \geq t_{0}
$$

Remark 2. Even when $A$ is linear, such a result is not quite obvious. Nevertheless, if $\mu(t) \rightarrow 0$ as $t \rightarrow+\infty$, the result is clear when $A$ is linear.

Proof of Theorem 1. The nonlinear semigroup $e^{-A t}$ being uniformly stable, there exist constants $M_{1}, \omega_{1}>0$ such that $\left\|e^{-A t} x^{0}\right\| \leq M_{1} e^{-\omega_{1} t}\left\|x^{0}\right\|$. Let $\delta>0$ and $t_{k}=t_{0}+k \delta$. Since the operator $A$ is accretive, the Bénilan's inequalities give for any $t \in\left[t_{k-1}, t_{k}\right]$,

$$
\left\|x(t)-e^{-\left(t-t_{k-1}\right) A} x\left(t_{k-1}\right)\right\| \leq \int_{t_{k-1}}^{t_{k}}\|B(\tau, x(\tau))\| d \tau .
$$

Thus, for $t \in\left[t_{k-1}, t_{k}\right]$, we have

$$
\begin{aligned}
\|x(t)\| & \leq\left\|e^{-\left(t-t_{k-1}\right) A} x\left(t_{k-1}\right)\right\|+\int_{t_{k-1}}^{t_{k}}\|B(\tau, x(\tau))\| d \tau \\
& \leq M_{1} e^{-\omega_{1}\left(t-t_{k-1}\right)}\left\|x\left(t_{k-1}\right)\right\|+\int_{t_{k-1}}^{t_{k}} \mu(\tau)\|x(\tau)\| d \tau .
\end{aligned}
$$

For $t \in\left[t_{k-1}, t_{k}\right]$, set

$$
y_{k}(t)=e^{\omega_{1}\left(t-t_{k-1}\right)}\|x(t)\|
$$

Then, for $t \in\left[t_{k-1}, t_{k}\right],(2.10)$ gives,

$$
y_{k}(t) \leq M_{1} y_{k}\left(t_{k-1}\right)+e^{\omega_{1} \delta} \int_{t_{k-1}}^{t} \mu(\tau) y_{k}(\tau) d \tau .
$$

Applying the Gronwall's formula, we get

$$
y_{k}(t) \leq M_{1} y_{k}\left(t_{k-1}\right) \exp \left(e^{\omega_{1} \delta} \int_{t_{k-1}}^{t} \mu(\tau) d \tau\right), t \in\left[t_{k-1}, t_{k}\right],
$$

and in particular

$$
y_{k}\left(t_{k}\right) \leq M_{1} e^{\alpha_{k}} y_{k}\left(t_{k-1}\right),
$$

where

$$
\alpha_{j}=e^{\omega_{1} \delta} \int_{t_{j-1}}^{t_{j}} \mu(\tau) d \tau, \quad j \in \mathbb{N}^{*}
$$


This implies, together with (2.11),

$$
\left\|x\left(t_{k}\right)\right\| \leq M_{1}^{k} \exp \left(-k \omega_{1} \delta+\sum_{j=1}^{j=k} \alpha_{j}\right)\left\|x\left(t_{0}\right)\right\| .
$$

Since we have

$$
\sum_{j=1}^{j=k} \alpha_{j}=e^{\omega_{1} \delta} \int_{t_{0}}^{t_{k}} \mu(\tau) d \tau
$$

the inequality (2.14) becomes

$$
\left\|x\left(t_{k}\right)\right\| \leq \exp \left(k \ln M_{1}-k \omega_{1} \delta+e^{\omega_{1} \delta} \int_{t_{0}}^{t_{k}} \mu(\tau) d \tau\right)\left\|x\left(t_{0}\right)\right\| .
$$

Now, take a positive constant $\omega_{0}$ such that $\omega_{0}>\max \left(1, \omega_{1}\right)$. Next, according to hypothesis $(\mathbf{H V})$, choose $\delta$ and then $t_{0}$ as follows:

$$
\left\{\begin{array}{l}
\delta>\frac{1}{\omega_{1}}\left(\ln M_{1}+2 \omega_{0}\right) \\
\int_{\xi}^{\xi+\delta} \mu(\tau) d \tau \leq \frac{\omega_{0}}{e^{\omega_{0} \delta}}, \forall \xi \geq t_{0}
\end{array}\right.
$$

It comes from (2.16)

$$
\alpha_{j} \leq e^{\omega_{1} \delta} \frac{\omega_{0}}{e^{\omega_{0} \delta}} \leq \omega_{0},
$$

for $j \in \mathbb{N}^{*}$. According to $(2.15,2.16)$ and $(2.17)$, we get

$$
\left\|x\left(t_{k}\right)\right\| \leq \exp \left(-\omega_{0}\left(\frac{t_{k}-t_{0}}{\delta}\right)\right)\left\|x\left(t_{0}\right)\right\| .
$$

One can show that (2.11) and (2.12) (with $k+1$ instead of $k$ ) give

$$
\|x(t)\| \leq M_{1} e^{-\omega_{1}\left(t-t_{k}\right)} e^{\alpha_{k+1}}\left\|x\left(t_{k}\right)\right\|,
$$

for all $t \in\left[t_{k}, t_{k+1}\right]$. Then, combining this last inequality with (2.18), we obtain

$$
\|x(t)\| \leq M_{1} e^{-\omega_{1}\left(t-t_{k-1}\right)+\alpha_{k+1}} \exp \left(-\omega_{0}\left(\frac{t_{k}-t_{0}}{\delta}\right)\right)\left\|x\left(t_{0}\right)\right\|, \forall t \in\left[t_{k}, t_{k+1}\right] .
$$

We deduce from (2.17) and (2.19) that for any $t \in\left[t_{k}, t_{k+1}\right]$, we have

$$
\begin{aligned}
\|x(t)\| & \leq M_{1} e^{-\omega_{1}\left(t-t_{k}\right)} e^{\omega_{0}} \exp \left(-\omega_{0} \frac{\left(t_{k}-t_{0}\right)}{\delta}\right)\left\|x\left(t_{0}\right)\right\| \\
& \leq M_{1} e^{\omega_{0}} e^{\omega_{0} \frac{t_{0}}{\delta}} e^{\frac{\omega_{0}\left(t-t_{k}\right)}{\delta}} e^{-\omega_{0} \frac{t}{\delta}}\left\|x\left(t_{0}\right)\right\| \\
& \leq M_{1} e^{-\omega_{0} \frac{t}{\delta}} e^{\omega_{0}\left(2+\frac{t_{0}}{\delta}\right)}\left\|x\left(t_{0}\right)\right\| .
\end{aligned}
$$

Hence, the required estimate of Theorem 1 follows. 


\section{Stability of a Rotating Body-BeAm System}

This section deals with exponential stability of the body-beam system (1.1-1.2) under suitable conditions on the nonlinear controls $f, g$ and $\gamma$.

\subsection{Hypotheses, notations and main result}

In the sequel, assume that the following hypotheses are satisfied:

F1) The functions $f$ and $g$ are in $\left\{h \in C^{0}(\mathbb{R}) ; h\right.$ is increasing; $\left.h(0)=0\right\}$.

F2) There exist constants $L_{1} \geq 0, L_{2}>0, L_{3}>0$ such that

$$
|f(x)| \leq L_{1}|x| \text { and } L_{2}|x| \leq|g(x)| \leq L_{3}|x|, \quad \forall x \in \mathbb{R}
$$

F3) The function $\gamma$ is Lipschitz on each bounded subset of $\mathbb{R}$ and for some $L_{4}>0$,

$$
\gamma(x) x \geq 0,|\gamma(x)| \geq L_{4}|x|, \forall x \in \mathbb{R} .
$$

The conditions F1) F2) ensure in particular the presence of at least a control force in the flexible beam because $g$ is not identically zero. In the other hand, the hypothesis F2) and the second condition of F3) are not deprived of practical interest. Indeed, in control system only a constrained input control is frequently available [1]. Usually, this can be satisfied by assuming hypotheses as F2) and F3) which are used in different previous works for nonlinear boundary stabilization problems (see for instance $[8,14]$ and [15]). In particular, F2), F3) allow the choice of a limited command for the part concerning $f$ and $\gamma$. Moreover, Hypotheses F1), F2) and F3) are obviously satisfied when $f, g$ and $\gamma$ are linear (as supposed in [16] for instance).

The closed loop system (1.1-1.2) can be written as follows:

$$
\left\{\begin{array}{l}
\rho y_{t t}+E I y_{x x x x}=\rho \omega^{2}(t) y \\
y(0, t)=y_{x}(0, t)=0 \\
y_{x x}(1, t)=-f\left(y_{t x}(1, t)\right) \\
y_{x x x}(1, t)=g\left(y_{t}(1, t)\right) \\
\dot{\omega}(t)=\frac{-\gamma\left(\omega(t)-\omega^{*}\right)-2 \rho \omega(t) \int_{0}^{1} y(t) y_{t}(t) d x}{I_{d}+\rho \int_{0}^{1} y(t)^{2} d x}
\end{array}\right.
$$

For the phase space of the system, take the real Hilbert space

$$
X=\mathcal{H} \times \mathbb{R}=H_{0}^{2} \times L^{2}(0,1) \times \mathbb{R},
$$

equipped with the inner product

$$
\langle(u, v, \xi),(\tilde{u}, \tilde{v}, \tilde{\xi})\rangle_{X}=\langle(u, v),(\tilde{u}, \tilde{v})\rangle_{\mathcal{H}}+\xi \tilde{\xi},
$$

where for $n \in \mathbb{N}$,

$$
H_{0}^{n}=\left\{u \in H^{n}(0,1) ; u(0)=u_{x}(0)=0\right\},
$$

and the space $\mathcal{H}$ is endoweded with the inner product

$$
\langle(u, v),(\tilde{u}, \tilde{v})\rangle_{\mathcal{H}}=\int_{0}^{1}\left(E I u_{x x} \tilde{u}_{x x}-\rho \omega^{* 2} u \tilde{u}+\rho v \tilde{v}\right) d x
$$


Note that the norm induced by this scalar product is equivalent to the usual one of the Hilbert space $H^{2}(0,1) \times$ $L^{2}(0,1)$ provided that $\left|\omega^{*}\right|<\sqrt{9 E I / \rho}$ (see [16] for details).

Set $\Phi=(y, z)$. Then, the system (3.1) can be written as follows

$$
\left\{\begin{array}{l}
\dot{\Phi}(t)+\tilde{A} \Phi(t)+\left(0,\left(\omega^{* 2}-\omega^{2}(t)\right) y(t)\right)=0 \\
\dot{\omega}(t)=\frac{-\gamma\left(\omega(t)-\omega^{*}\right)-2 \rho \omega(t)<y(t), z(t)>_{L^{2}(0,1)}}{I_{d}+\rho\|y(t)\|_{L^{2}(0,1)}^{2}}
\end{array}\right.
$$

where the nonlinear operator $\tilde{A}$ is defined by

$$
\mathcal{D}(\tilde{A})=\left\{(y, z) \in H_{0}^{4} \times H_{0}^{2} ; \begin{array}{l}
y_{x x}(1)=-f\left(z_{x}(1)\right), \\
y_{x x x}(1)=g(z(1))
\end{array}\right\},
$$

and

$$
\tilde{A}(y, z)=\left(-z, \frac{E I}{\rho} y_{x x x x}-\omega^{* 2} y\right) .
$$

Clearly, the system (3.2) is equivalent to the following one

$$
(\dot{\Phi}(t), \dot{\omega}(t))+(A+B)(\Phi(t), \omega(t))=0
$$

where

$$
\left\{\begin{array}{l}
A(\Phi, \omega)=(\tilde{A} \Phi, 0), \text { with } \mathcal{D}(A)=\mathcal{D}(\tilde{A}) \times \mathbb{R} \\
B(\Phi, \omega)=\left(0,\left(\omega^{* 2}-\omega^{2}\right) y, \frac{-\gamma\left(\omega-\omega^{*}\right)-2 \rho \omega<y, z>_{L^{2}(0,1)}}{I_{d}+\rho\|y\|_{L^{2}(0,1)}^{2}}\right) .
\end{array}\right.
$$

We will show in the sequel that (3.5) is in fact a formulation of the closed loop system (1.1-1.2) in a $C P$ form.

The main result of this section is:

Theorem 2. Suppose that $\left|\omega^{*}\right|<\sqrt{9 E I / \rho}$. Then under hypotheses F1), F2) and F3), for each initial data $\left(\Phi_{0}, \omega_{0}\right) \in \mathcal{D}(A)$ the solution $(\Phi(t), \omega(t))$ of the closed-loop system (3.5) tends exponentially to $\left(0, \omega^{*}\right)$ in $X$ as $t \rightarrow+\infty$.

First, we deal with the existence and uniqueness of the solution of the following subsystem

$$
\left\{\begin{array}{l}
\dot{\Phi}(t)+\tilde{A} \Phi(t)=0 \\
\Phi(0)=\Phi_{0}
\end{array}\right.
$$

where $\tilde{A}$ is the nonlinear operator defined by $(3.3-3.4)$.

\subsection{Wellposedness of the subsystem (3.7)}

We have the following proposition.

Proposition 2. Assume that $\left|\omega^{*}\right|<\sqrt{9 E I / \rho}$. The operator $\tilde{A}$ defined by (3.3-3.4) is m-accretive in $\mathcal{H}$ with dense domain. 
Proof of Proposition 2. A straightforward computation shows that for all $(y, z),(\tilde{y}, \tilde{z})$ in $\mathcal{D}(\tilde{A})$,

$$
\begin{aligned}
\langle\tilde{A}(y, z)-\tilde{A}(\tilde{y}, \tilde{z}),(y, z)-(\tilde{y}, \tilde{z})\rangle_{\mathcal{H}}= & E I(z(1)-\tilde{z}(1))(g(z(1))-g(\tilde{z}(1))) \\
& +E I\left(z_{x}(1)-\tilde{z}_{x}(1)\right)\left(f\left(z_{x}(1)\right)-f\left(\tilde{z}_{x}(1)\right)\right) .
\end{aligned}
$$

Using Hypothesis F1), we deduce that $\langle\tilde{A}(y, z)-\tilde{A}(\tilde{y}, \tilde{z}),(y, z)-(\tilde{y}, \tilde{z})\rangle_{\mathcal{H}} \geq 0$ and thus the operator $\tilde{A}$ is accretive.

Now, we have to show the maximality of $\tilde{A}$, what amounts to saying that (see [6]), for any given $(u, v) \in$ $\mathcal{H}=H_{0}^{2} \times L^{2}(0,1)$, there exists $(y, z) \in \mathcal{D}(\tilde{A})$ such that $(I+\tilde{A})(y, z)=(u, v)$. Equivalently, we seek $y$ and $z$ satisfying

$$
\left\{\begin{array}{l}
y-z=u \\
z-\omega^{* 2} y+\frac{E I}{\rho} y_{x x x x}=v \\
y_{x x}(1)+f\left(z_{x}(1)\right)=0 \\
y_{x x x}(1)-g(z(1))=0 \\
y \in H_{0}^{4}, z \in H_{0}^{2}
\end{array}\right.
$$

Eliminating the unknown $z$ in (3.9), one obtains

$$
\left\{\begin{array}{l}
E I y_{x x x x}+\rho\left(1-\omega^{* 2}\right) y=\rho(u+v) \\
y_{x x}(1)+f\left(y_{x}(1)-u_{x}(1)\right)=0 \\
y_{x x x}(1)-g(y(1)-u(1))=0 \\
y \in H_{0}^{4}
\end{array}\right.
$$

Let us define, as in [22], the function $J(\cdot)$ on $H_{0}^{2}$ by

$$
J(\psi)=\frac{1}{2}\left\{\int_{0}^{1}\left[E I \psi_{x x}^{2}+\rho\left(1-\omega^{* 2}\right) \psi^{2}\right] d x\right\}-\rho \int_{0}^{1}(u+v) \psi d x+E I\left[F\left(\psi_{x}(1)-u_{x}(1)\right)+G(\psi(1)-u(1))\right],
$$

where

$$
F(x)=\int_{0}^{x} f(\xi) d \xi, \quad G(x)=\int_{0}^{x} g(\xi) d \xi, \quad \forall x \in \mathbb{R}
$$

From Hypothesis F1), we deduce that $F$ and $G$ are continuously differentiable on $\mathbb{R}$, convex and $F(x) \geq$ $0, G(x) \geq 0$ for all $x \in \mathbb{R}$. Consequently, we can claim that $J$ is convex, coercive and continuous in $H_{0}^{2}$. Hence, by a minimization theorem (Prop. 38.15, [25] p. 155), there exists a function $y \in H_{0}^{2}$ such that $J(y)=\inf _{\psi \in H_{0}^{2}} J(\psi)$. This implies that the function

$$
\Theta: \lambda \longmapsto \Theta(\lambda)=J(y+\lambda \psi)
$$


admits a minimum at $\lambda=0$ and thus $\left.\frac{d}{d \lambda}(J(y+\lambda \psi))\right|_{\lambda=0}=0, \forall \psi \in H_{0}^{2}$. This means, thanks to a direct computation, that for any $\psi \in H_{0}^{2}$, we have

$$
\begin{aligned}
\int_{0}^{1} y_{x x} \psi_{x x} d x= & \int_{0}^{1}\left[\rho\left(\omega^{* 2}-1\right) y+\rho(u+v)\right] \psi d x+g(y(1)-u(1)) \psi(1) \\
& +E \operatorname{If}\left(y_{x}(1)-u_{x}(1)\right) \psi_{x}(1) .
\end{aligned}
$$

Integrating by parts in the last relation, we can prove after a careful computation that $y \in H_{0}^{4}$ and

$$
\left\{\begin{array}{l}
E I y_{x x x x}+\rho\left(1-\omega^{* 2}\right) y=\rho(u+v), \\
y_{x x}(1)+f\left(y_{x}(1)-u_{x}(1)\right)=0 \\
y_{x x x}(1)-g(y(1)-u(1))=0
\end{array}\right.
$$

We deduce that $y$ is solution of the system (3.10). Then $(y, y-u)$ satisfies clearly system (3.9) and thus the maximality of $\tilde{A}$ is proved.

Finally, it remains to prove the density of the domain $\mathcal{D}(\tilde{A})$.

Lemma 4. The domain $\mathcal{D}(\tilde{A})$ of $\tilde{A}$ is dense in $\mathcal{H}$.

The proof of this Lemma propose a general approach for the density of the domain of nonlinear m-accretive operators on uniformly convex spaces when the nonlinearity is concentrated in the domain. This result supplies the well-known analogous theorem for linear m-accretive operators on reflexive Banach spaces (see $[4,20]$ ).

Proof of Lemma 4. Because of $\tilde{A}$ is maximal, there exits $\left(u^{n}, v^{n}\right) \in \mathcal{D}(\tilde{A})$ such that

$$
\left(u^{n}, v^{n}\right)=\left(I+\lambda_{n} \tilde{A}\right)^{-1}\left(u^{0}, v^{0}\right) .
$$

We want to prove

$$
\left(u^{n}, v^{n}\right) \rightarrow\left(u^{0}, v^{0}\right) \quad \text { in } \mathcal{H} .
$$

Clearly, this implies the density of $\mathcal{D}(\tilde{A})$. Since $\tilde{A}$ is m-accretive in the Hilbert space, the resolvent operator $\left(I+\lambda_{n} \tilde{A}\right)^{-1}$ is nonexpansive, whence

$$
\left\|\left(u^{n}, v^{n}\right)\right\|_{\mathcal{H}} \leq\left\|\left(u^{0}, v^{0}\right)\right\|_{\mathcal{H}} .
$$

Then the bounded subset $\left\{\left(u^{n}, v^{n}\right)\right\}_{n}$ is weakly precompact in $\mathcal{H}$ and thus there exists a subsequence, still indexed by $n$ for convenience, such that

$$
\left\{\begin{array}{l}
\left(u_{x x}^{n}\right)_{n} \rightarrow\left(u_{\infty}\right)_{x x} \\
\left(v^{n}\right)_{n} \rightarrow v_{\infty}
\end{array}\right.
$$

weakly in $L^{2}(0,1)$. But, from (3.13) it follows

$$
\left\{\begin{array}{l}
u^{n}-\lambda_{n} v^{n}=u^{0}, \\
v^{n}+\lambda_{n} \frac{E I}{\rho} u_{x x x x}^{n}-\omega^{* 2} \lambda_{n} u^{n}=v^{0} .
\end{array}\right.
$$

Then, it is easy to show that (3.17) implies $\left(u_{x x}^{n}\right)_{n} \rightarrow u_{x x}^{0}$ and $\left(v^{n}\right)_{n} \rightarrow v^{0}$ in $\mathcal{D}^{\prime}(0,1)$ (i.e. in the sense of distributions). This together with (3.16) implies that $\left(u_{\infty}\right)_{x x}=u_{x x}^{0}$ and $v_{\infty}=v^{0}$ in $\mathcal{D}^{\prime}(0,1)$. Finally, a 
classical property of Lebesgue spaces (see [7] Lem. IV.2, p. 61) gives $\left(u_{\infty}\right)_{x x}=u_{x x}^{0}$ and $v_{\infty}=v^{0}$ in $L^{2}(0,1)$. Consequently (3.16) becomes

$$
\left(u^{n}, v^{n}\right)_{n} \rightarrow\left(u^{0}, v^{0}\right)
$$

weakly in $\mathcal{H}(0,1)$. Now, since $\mathcal{H}$ is a Hilbert space and a fortiori a uniformly convex space, from (3.15) and (3.18) we deduce $\left(u^{n}, v^{n}\right)_{n} \rightarrow\left(u^{0}, v^{0}\right)$ strongly in $\mathcal{H}(0,1)$ (see for instance [7], Prop. III.30, p.521). This ends the proof of Lemma 4.

We have thus shown Proposition 2.

The following lemma is a consequence of Proposition 2 and well known results in [6].

Lemma 5. i) For any initial data $\Phi_{0}=\left(y_{0}, z_{0}\right) \in \mathcal{D}(\tilde{A})$, the system (3.7) admits a unique solution $\Phi(t)=$ $(y(t), z(t)) \in \mathcal{D}(\tilde{A})$ such that

$$
(y, z) \in L^{\infty}\left(\mathbb{R}^{+} ; \mathcal{H}\right) \text {, and } \frac{d}{d t}(y, z) \in L^{\infty}\left(\mathbb{R}^{+} ; \mathcal{H}\right)
$$

The solution $\Phi=(y, z)$ is given by $\Phi(t)=e^{-\tilde{A} t} \Phi_{0}$, for all $t \geq 0$ where $e^{-\tilde{A} t}$ is the semigroup generated by $-\tilde{A}$ on $\overline{\mathcal{D}(\tilde{A})}=\mathcal{H}$. Moreover, the function $t \longmapsto\|\tilde{A} \Phi(t)\|_{\mathcal{H}}$ is decreasing.

ii) For any initial data $\Phi_{0}=\left(y_{0}, z_{0}\right) \in \overline{\mathcal{D}(\tilde{A})}=\mathcal{H}$, the equation (3.7) admits a unique mild solution $\Phi(t)=$ $e^{-\tilde{A} t} \Phi_{0}$ which is bounded on $\mathbb{R}^{+}$by $\left\|\Phi_{0}\right\|_{\mathcal{H}}$ and

$$
\Phi=(y, z) \in C^{0}\left(\mathbb{R}^{+} ; \mathcal{H}\right) .
$$

\subsection{Asymptotic stability of $e^{-\tilde{A} t}$}

In this subsection, using the LaSalle's invariance principle [11-13], we show that the nonlinear semigroup $e^{-\tilde{A} t}$ is asymptotically stable.

Proposition 3. Assume that $\left|\omega^{*}\right|<\sqrt{9 E I / \rho}$. The semigroup $e^{-\tilde{A} t}$ is asymptotically stable in $\mathcal{H}$, i.e., for any initial data $\Phi_{0}=\left(y_{0}, z_{0}\right) \in \overline{\mathcal{D}(\tilde{A})}=\mathcal{H}$, we have $e^{-\tilde{A} t} \Phi_{0} \rightarrow 0$ as $t \rightarrow+\infty$.

Proof of Proposition 3. Since the nonlinear semigroup $e^{-\tilde{A} t}$ is contractive and since $\mathcal{D}(\tilde{A})$ is dense in $\mathcal{H}$, it suffices to prove the Proposition for any initial data $\Phi_{0}=\left(y_{0}, z_{0}\right) \in \mathcal{D}(\tilde{A})$. Let $\Phi_{0}=\left(y_{0}, z_{0}\right) \in \mathcal{D}(\tilde{A})$. By virtue of Lemma 5 , we know that the trajectory $\left\{e^{-\tilde{A} t} \Phi_{0} ; t \geq 0\right\}$ is a bounded set for the graph norm. Furthermore, one can show directly that the injection

$$
i:\left(\mathcal{D}(\tilde{A}),\|\|_{\mathcal{D}(\tilde{A})}\right) \longrightarrow \mathcal{H}
$$

is compact. This implies that the considered trajectory is precompact in $\mathcal{H}$. Applying the LaSalles's invariance principle $[11,13]$, we deduce that the $\omega$-limit set

$$
\omega\left(\Phi_{0}\right)=\left\{\left(\psi_{1}, \psi_{2}\right) \in \mathcal{H} ;\left(\psi_{1}, \psi_{2}\right)=\lim _{n \rightarrow \infty} S\left(t_{n}\right)\left(\Phi_{0}\right) \text { with } t_{n} \rightarrow \infty \text { as } n \rightarrow \infty\right\}
$$

is non empty, compact, invariant under the semigroup $e^{-\tilde{A} t}$ and

$$
e^{-\tilde{A} t} \Phi_{0} \longrightarrow \omega\left(\Phi_{0}\right) \quad \text { as } t \rightarrow+\infty .
$$


Moreover, we deduce from the m-accretivity of $\tilde{A}$ (see [6])

$$
\omega\left(\Phi_{0}\right) \subset \mathcal{D}(\tilde{A}) .
$$

In order to prove the asymptotic stability, it is sufficient to show that the $\omega$-limit set $\omega\left(\Phi_{0}\right)$ reduces to $\{0\}$. For this, let $\left(\tilde{\phi}_{1}, \tilde{\phi}_{2}\right) \in \omega\left(\Phi_{0}\right)$ and

$$
(\tilde{y}, \tilde{z})(t)=e^{-\tilde{A} t}\left(\tilde{\phi}_{1}, \tilde{\phi}_{2}\right) \subset \omega\left(\Phi_{0}\right) \subset \mathcal{D}(\tilde{A}), \forall t \geq 0 .
$$

But, $\|(\tilde{y}, \tilde{z})(t)\|_{\mathcal{H}}$ is constant on $\mathbb{R}^{+}$(see [12]) and thus $\frac{d}{d t}\|(\tilde{y}, \tilde{z})(t)\|_{\mathcal{H}}^{2}=0$, on $\mathbb{R}^{+}$. Since $(\tilde{y}, \tilde{z})$ is a strong solution, this means

$$
\langle\tilde{A}(\tilde{y}, \tilde{z})(t),(\tilde{y}, \tilde{z})(t)\rangle_{\mathcal{H}}=0 \text {, a.e. } t \geq 0 \text {. }
$$

Now, from (3.8) and (3.19), it follows

$$
\langle\tilde{A}(\tilde{y}, \tilde{z})(t),(\tilde{y}, \tilde{z})(t)\rangle_{\mathcal{H}}=E I \tilde{z}(1, t) g(\tilde{z}(1, t))+E I \tilde{z}_{x}(1, t) f\left(\tilde{z}_{x}(1, t)\right)=0 \text {, a.e. } t \geq 0 .
$$

We deduce from (3.20) and F1) that $\tilde{y}$ is the strong solution on $\mathbb{R}^{+}$of the following linear system

$$
\left\{\begin{array}{l}
\rho y_{t t}+E I y_{x x x x}=\rho \omega^{* 2} y \\
y(0, t)=y_{x}(0, t)=0 \\
y_{x x}(1, t)=0 \\
y_{x x x}(1, t)=y_{t}(1, t)=0, \\
\left.\left(y(0), y_{t}(0)\right)=\left(\tilde{\phi}_{1}, \tilde{\phi}_{2}\right) \in \omega\left(\Phi_{0}\right)\right)
\end{array}\right.
$$

However, it was shown in [16] that zero is the unique (mild) solution of (3.21). Of course, for such a linear system the notions of mild, strong or classical solutions coincide. Therefore, the proof of Proposition 3 is complete.

Remark 3. Notice that the well-posedness result as well as the asymptotic stability one is obtained with only Hypothesis F1).

\subsection{Exponential stability of $e^{-\tilde{A} t}$}

In this subsection, by using the multiplier method (see [15]), we obtain an exponential stability result of the semigroup $e^{-\tilde{A} t}$ under hypotheses F1) and F2).

Proposition 4. Assume that $\left|\omega^{*}\right|<\sqrt{9 E I / \rho}$. Let $\Phi=(y, z)$ be the solution of (3.7) stemmed from $\Phi_{0}=$ $\left(y_{0}, z_{0}\right) \in \overline{\mathcal{D}(\tilde{A})}=\mathcal{H}$. If hypotheses F1) and F2) are satisfied, then there exist (uniform) constants $\tilde{M}, \tilde{\mu}>0$ such that

$$
\| \Phi(t))\left\|_{\mathcal{H}} \leq \tilde{M} e^{-\tilde{\mu} t}\right\| \Phi_{0} \|_{\mathcal{H}}, \quad \forall t \geq 0 .
$$

Proof of proposition 4. As in the proof of Proposition 3, we may assume that $\Phi_{0}=\left(y_{0}, z_{0}\right) \in \mathcal{D}(\tilde{A})$. Then, let us write $\Phi(t)=e^{-\tilde{A} t} \Phi_{0}=\left(y, y_{t}\right)(t)$ and define the functional $\xi$ as

$$
\xi(t)=2 \int_{0}^{1} x y_{t} y_{x} d x, \quad t \geq 0
$$


Returning to the definition of mild solutions, we note that the function $t \mapsto \xi(t)$ is everywhere defined and continuous on $\left[0,+\infty\left[\right.\right.$ (even without assuming $\left.\left(y_{0}, z_{0}\right) \in \mathcal{D}(\tilde{A})\right)$. Standard computations (see (3.27) below) provide a constant $K_{1}$ such that

$$
|\xi(t)| \leq K_{1}\|(y(t), z(t))\|_{\mathcal{H}}^{2}, \quad \forall t \geq 0 .
$$

On the other hand, according to Lemma 5 and (3.1), a straightforward computation gives

$$
\begin{aligned}
\xi_{t}(t)= & -2 \frac{E I}{\rho} y_{x}(1, t) f\left(y_{x t}(1, t)\right)-2 \frac{E I}{\rho} y_{x}(1, t) g\left(y_{t}(1, t)\right) \\
& +y_{t}^{2}(1, t)+\frac{E I}{\rho} f^{2}\left(y_{x t}(1, t)\right)+\omega^{* 2} y^{2}(1, t)-\int_{0}^{1}\left[y_{t}^{2}+3 \frac{E I}{\rho} y_{x x}^{2}+\omega^{* 2} y^{2}\right] d x,
\end{aligned}
$$

a.e. $t \geq 0$.

Moreover, using once again the Cauchy-Schwarz's inequality, we have

$$
\left\{\begin{aligned}
-2 \frac{E I}{\rho} y_{x}(1, t) g\left(y_{t}(1, t)\right) & \leq \frac{E I}{\rho \theta} y_{x}^{2}(1, t)+\theta \frac{E I}{\rho} g^{2}\left(y_{t}(1, t)\right), \\
-2 \frac{E I}{\rho} y_{x}(1, t) f\left(y_{x t}(1, t)\right) & \leq \frac{E I}{\rho \theta} y_{x}^{2}(1, t)+\theta \frac{E I}{\rho} f^{2}\left(y_{x t}(1, t)\right),
\end{aligned}\right.
$$

for any $\theta>0$.

From $y(0)=y_{x}(0)=0$, it easily follows

$$
y_{x}^{2}(1, t) \leq \int_{0}^{1} y_{x x}^{2} d x, \quad \text { and } \quad \omega^{* 2} y^{2}(1, t) \leq \frac{\omega^{* 2}}{3} \int_{0}^{1} y_{x x}^{2} d x .
$$

The first inequality of (3.27), together with (3.26), implies

$$
-2 \frac{E I}{\rho} y_{x}(1, t) g\left(y_{t}(1, t)\right)-2 \frac{E I}{\rho} y_{x}(1, t) f\left(y_{x t}(1, t)\right) \leq \theta \frac{E I}{\rho} g^{2}\left(y_{t}(1, t)\right)+\theta \frac{E I}{\rho} f^{2}\left(y_{x t}(1, t)\right)+2 \frac{E I}{\rho \theta} \int_{0}^{1} y_{x x}^{2} d x .
$$

Combining (3.25), the second inequality of (3.27) and (3.27), we get

$$
\begin{aligned}
\xi_{t}(t) \leq & y_{t}^{2}(1, t)+\theta \frac{E I}{\rho} g^{2}\left(y_{t}(1, t)\right)+(1+\theta) \frac{E I}{\rho} f^{2}\left(y_{x t}(1, t)\right) \\
& +\frac{1}{\rho} \int_{0}^{1}\left[-\rho y_{t}^{2}+\left(\frac{2}{\theta}+\frac{\rho \omega^{* 2}}{3 E I}-3\right) E I y_{x x}^{2}-\rho \omega^{* 2} y^{2}\right] d x,
\end{aligned}
$$

a.e. $t \geq 0$.

Taking into account the condition $\left|\omega^{*}\right|<\sqrt{9 E I / \rho}$, we can choose $\theta$ in order to have

$$
\frac{2}{\theta}+\frac{\rho \omega^{* 2}}{3 E I}-3<0
$$


Thus remembering the definition of the norm $\|\cdot\|_{\mathcal{H}}$, we find a constant $K_{2}>0$ satisfying

$$
\xi_{t}(t) \leq y_{t}^{2}(1, t)+\theta \frac{E I}{\rho} g^{2}\left(y_{t}(1, t)\right)+(1+\theta) \frac{E I}{\rho} f^{2}\left(y_{x t}(1, t)\right)-K_{2}\|(y(t), z(t))\|_{\mathcal{H}}^{2},
$$

a.e. $t \geq 0$.

Given $\epsilon>0$, we introduce (see [8] and [22]) the perturbed energy by

$$
E^{\epsilon}(t)=E_{1}(t)+\epsilon \xi(t),
$$

where

for all $t \geq 0$.

$$
E_{1}(t)=\frac{1}{2}\|(y(t), z(t))\|_{\mathcal{H}}^{2}
$$

The energy $E_{1}(t)$ is a decreasing function: more precisely, owing to the accretivity of $\tilde{A}$, we have

$$
E_{1 t}(t)=-E I y_{t}(1, t) g\left(y_{t}(1, t)\right)-E I y_{x t}(1, t) f\left(y_{x t}(1, t)\right) \text { a.e } t \geq 0 .
$$

But (3.23) and (3.31) imply that for all $t \geq 0$, we have

$$
K_{3}{ }^{-1} E^{\epsilon}(t) \leq E_{1}(t) \leq K_{3} E^{\epsilon}(t)
$$

provided $K_{3}>1$ and $\epsilon \leq \frac{1}{2}\left(1-K_{3}{ }^{-1}\right)$.

Combining (3.31) and (3.30), we obtain

$$
E_{t}^{\epsilon}(t) \leq E_{1 t}(t)+\epsilon y_{t}^{2}(1, t)+\epsilon \theta \frac{E I}{\rho} g^{2}\left(y_{t}(1, t)\right)+\epsilon(1+\theta) \frac{E I}{\rho} f^{2}\left(y_{x t}(1, t)\right)-2 \epsilon K_{2} E_{1}(t),
$$

a.e. $t \geq 0$. In addition, hypothesis $\mathrm{F} 2$ ) leads us to

$$
\left\{\begin{array}{l}
y_{t}^{2}(1, t)+\theta \frac{E I}{\rho} g^{2}\left(y_{t}(1, t)\right) \leq\left(\frac{1}{L_{2}}+\theta L_{3} \frac{E I}{\rho}\right) y_{t}(1, t) g\left(y_{t}(1, t)\right) \\
(1+\theta) \frac{E I}{\rho} f^{2}\left(y_{x t}(1, t)\right) \leq(1+\theta) \frac{E I}{\rho} L_{1} y_{x t}(1, t) f\left(y_{x t}(1, t)\right) .
\end{array}\right.
$$

a.e. $t \geq 0$.

Plugging these two last inequalities into (3.34) and using (3.32), we get

$$
\begin{aligned}
E_{t}^{\epsilon}(t) & \leq-2 \epsilon K_{2} E_{1}(t)+\left[\epsilon\left(\frac{1}{L_{2}}+\theta L_{3} \frac{E I}{\rho}\right)-E I\right] y_{t}(1, t) g\left(y_{t}(1, t)\right) \\
& +\left[\epsilon L_{1}(1+\theta) \frac{E I}{\rho}-E I\right] y_{x t}(1, t) f\left(y_{x t}(1, t)\right) .
\end{aligned}
$$

This implies

$$
E_{t}^{\epsilon}(t) \leq-2 K_{2} \epsilon E_{1}(t)
$$

a.e. $t \geq 0$, provided that, $\epsilon$ satisfies

$$
\left\{\begin{array}{l}
\epsilon\left(\frac{1}{L_{2}}+\theta L_{3} \frac{E I}{\rho}\right)-E I \leq 0 \\
\epsilon L_{1}(1+\theta) \frac{E I}{\rho}-E I \leq 0
\end{array}\right.
$$


Finally, from (3.33) and (3.35), for such $\epsilon>0$ we deduce

$$
E_{t}^{\epsilon}(t) \leq-2 \epsilon K_{3}^{-1} K_{2} E^{\epsilon}(t)
$$

a.e. $t \geq 0$. Solving this differential inequality (remark that $E^{\epsilon}$ is absolutely continuous since $\Phi$ is locally Lipschitz) and using once again (3.33), we obtain the required exponential decay of $E_{1}(t)=\frac{1}{2}\|\Phi(t)\|_{\mathcal{H}}^{2}$ with $\tilde{\mu}=\epsilon K_{3}{ }^{-1} K_{2}$.

\subsection{The global system (3.5)}

a) Cauchy problem

By using Proposition 1, we will show that the global system (3.5) is well posed on [0, $\infty$. Indeed, we recall that the global system (3.5) can be written as an evolution equation

$$
\left(\begin{array}{c}
\dot{\Phi}(t) \\
\dot{\omega}(t)
\end{array}\right)+(A+B)\left(\begin{array}{c}
\Phi(t) \\
\omega(t)
\end{array}\right)=0
$$

where the operators $A$ and $B$ are defined in (3.6).

First, we have shown in Proposition 4 that the operator $\tilde{A}$ defined by $(3.3-3.4)$ is m-accretive in $\mathcal{H}$ with dense domain $\mathcal{D}(\tilde{A})$. We deduce thus, from (3.6), that the operator $A$ is also m-accretive with dense domain $\mathcal{D}(A)=\mathcal{D}(\tilde{A}) \times \mathbb{R}$ in $X=\mathcal{H} \times \mathbb{R}$.

Second, as the reader can easily see, the operator $B$, defined in (3.6), is Lipschitz on bounded subsets of $X$ and therefore $B$ satisfies hypothesis (HII) of Section 2. So $(3.5)$ has a local solution $(\Phi, \omega)(t)=(y, z, \omega)(t)$.

Consider now the function $V: X \rightarrow \mathbb{R}^{+}$defined by

$$
V\left(y_{1}, z_{1}, \omega_{1}\right)=\frac{1}{2} I_{d}\left(\omega_{1}-\omega^{*}\right)^{2}+\frac{1}{2}\left(\omega_{1}-\omega^{*}\right)^{2} \int_{0}^{1} \rho y_{1}^{2} d x+\frac{1}{2} \int_{0}^{1}\left(\rho z_{1}^{2}+E I y_{1 x x}^{2}\right) d x-\frac{1}{2} \omega^{* 2} \int_{0}^{1} \rho y_{1}^{2} d x
$$

for $\left(y_{1}, z_{1}, \omega_{1}\right) \in X$. We claim that this function is a reasonable choice of Lyapunov function for (3.5). Indeed, on the one hand, it is easy to check

$$
V\left(y_{1}, z_{1}, \omega_{1}\right) \geq K\left\|\left(y_{1}, z_{1}, \omega_{1}\right)\right\|_{X}^{2}
$$

for some positive constant $K$ and for all $\left(y_{1}, z_{1}, \omega_{1}\right) \in X$. On the other hand, from Lemma 3 , we know that each local solution of (3.5), with initial data in $\mathcal{D}(A)$, is a strong one. Moreover, a straightforward computation shows that for any initial condition $\left(y_{0}, z_{0}, \omega_{0}\right) \in \mathcal{D}(A)$, the corresponding strong solution $(y, z, \omega)(t)$ of $(3.5)$ satisfies

$$
\frac{d V}{d t}(\Phi, \omega)(t)=-E I\left[y_{x t}(1, t) f\left(y_{x t}(1, t)\right)+y_{t}(1, t) g\left(y_{t}(1, t)\right)\right]-\left(\omega(t)-\omega^{*}\right) \gamma\left(\omega(t)-\omega^{*}\right) \leq 0
$$

almost everywhere on the domain $\left[0, T_{\max }\right)$ of this local solution $(y, z, \omega)(t)$. Thus, the function $t \longmapsto V(y(t)$, $z(t), \omega(t))$ is non increasing on its domain. Then by Lemma 2 and (3.38), (HIII) holds for the system (3.5).

Now, from Proposition 1 we are in position to claim that for any initial condition $\left(y_{0}, z_{0}, \omega_{0}\right) \in X$, the global system $(3.5)$ has a unique solution $(\Phi, \omega)$ on $[0, \infty]$.

Proof of Theorem 2. Initially, we will restrict ourselves to the system

$$
\left\{\begin{array}{l}
\dot{\Phi}(t)+\tilde{A} \Phi(t)+\tilde{B}(t, \Phi(t))=0, \text { on }[0, \infty[ \\
\Phi(0)=\Phi_{0}
\end{array}\right.
$$


where $\Phi(t)=(y, z)(t), \Phi_{0} \in \mathcal{D}(\tilde{A})$ and

$$
\tilde{B}(t,(u, v))=\left(0,\left(\omega^{* 2}-\omega^{2}(t)\right) u\right)
$$

$\omega(t)$ being such that $(\Phi(t), \omega(t))$ is the solution of the global system $(3.5)$ with initial data $\left(\Phi_{0}, \omega_{0}\right) \in \mathcal{D}(A)$. From (3.40), we have

$$
\|\tilde{B}(t, \Phi(t))\|_{\mathcal{H}} \leq\left|\omega^{* 2}-\omega^{2}(t)\right|\|\Phi(t)\|_{\mathcal{H}} .
$$

In addition, one can show that $(3.37,3.39)$ and F3) give

$$
\omega^{*}-\omega(\cdot) \in L^{2}\left(\left[0, \infty[; \mathbb{R}) \cap L^{\infty}([0, \infty[; \mathbb{R}) .\right.\right.
$$

Consequently, (HV) holds with $\mu(t)=\left|\omega^{* 2}-\omega^{2}(t)\right|$. (Really, we have the stronger condition $\lim _{t \rightarrow+\infty} \omega(t)=\omega^{*}$ ). Therefore, all the assumptions of Theorem 1 are satisfied. Thus, there exist strictly positive constants $M$ and $\kappa$ such that

$$
\|\Phi(t)\|_{\mathcal{H}} \leq M e^{-\kappa t}, \forall t \geq 0 .
$$

Finally, returning to the second equation of (3.5) and using F3) we prove analogously to [16] that $\omega^{*}-\omega(\cdot)$ tends also exponentially to zero. The proof of Theorem 2 is now complete.

\section{REFERENCES}

[1] J. Ackermann, Sampled-data control system: Analysis and synthesis, robust system design, Springer-Verlag (1985).

[2] J. Baillieul and M. Levi, Rotational elastic dynamics. Physica D, 27 (1987) 43-62 .

[3] P. Bénilan, Équations d'évolution dans un espace de Banach quelconque et applications, Thèse, Paris XI, Orsay (1972).

[4] P. Bénilan, M.G. Crandal and A. Pazy, Nonlinear evolution equations in Banach spaces, monograph in preparation.

[5] A.M. Bloch and E.S. Titi, On the dynamics of rotating elastic beams, in Proc. Conf. New Trends Syst. theory, Genoa, Italy, July 9-11, 1990, Conte, Perdon, and Wyman, eds., Cambridge, MA: Birkhäuser (1990).

[6] H. Brezis, Opérateurs maximaux monotones et semi-groupes de contractions dans les espaces de Hilbert, North-Holland, Amsterdam, London (1973).

[7] H. Brezis, Analyse Fonctionnelle. Théorie et applications, Masson (1983).

[8] F. Conrad and B. Rao, Decay of solutions of the wave equation in a star-shaped domain with nonlinear boundary feedback. Asymptotic Analysis, 7 (1993) 159-177.

[9] J.-M. Coron and B. d'Andréa-Novel, Stabilization of a rotating body-beam without damping. IEEE Trans. Automat. Contr., 43 (1998) 608-618.

[10] M.G. Crandall, Nonlinear semigroups and evolution governed by accretive operators. Pro. Sympo. in pure Math. 45 (1986) 305-337.

[11] C.M. Dafermos and M. Slemrod, Asymptotic behaviour of non linear contractions semi-groups, J. Func. Anal . 14 (1973) 97-106.

[12] A. Haraux, Systèms Dynamique Dissipatifs et Applications. Collection RMA (17) Masson, Paris (1991).

[13] V. Jurdjevic and J. P. Quin, Controllability and stability, J. Differential Equations, 28 (1978) 381-389.

[14] V. Komornik and E. Zuazua, A direct method for the boundary stabilization of the wave equation. J. Math. Pures Appl. , 69 (1990) 33-54.

[15] V. Komornik, Exact Controllability and Stabilization. The Multiplier Method, Masson and John Wiley (1994).

[16] H. Laousy, C.Z. Xu and G. Sallet, Boundary feedback stabilization of a rotating body-beam system. IEEE Trans. Automat. Contr., 41 (1996) 241-245.

[17] O. Morgül, Orientation and stabilization of a flexible beam attached to a rigid body: Planar motion. IEEE Trans. Automat. Contr., 36 (1991) 953-963.

[18] O. Morgül, Constant angular velocity control of a rotating flexible structure, in Proc. 2nd Conf., ECC'93., Groningen, Netherlands (1993) 299-302.

[19] O. Morgül, Control of a rotating flexible structure. IEEE Trans. Automat. Contr. 39 (1994) 351-356.

[20] A. Pazy, Semigroups of Linear Operators and Applications to Partial Differential Equations. Springer Verlag, New York (1983). 
[21] M. Pierre, Perturbations localement lipschitziennes et continues d'opérateurs m-accretifs. Proc. Amer. Math. Soc., 58 (1976) 124-128.

[22] B. Rao, Decay estimate of solution for hybrid system of flexible structures. Euro. J. Appl. Math. 4 (1993) $303-319$.

[23] C.Z. Xu and J. Baillieul, Stabilizability and stabilization of a rotating body-beam system with torque control. IEEE Trans. Automat. Contr. 38 (1993) 1754-1765.

[24] C.Z. Xu and G. Sallet, Boundary stabilization of a rotating flexible system. Lecture Notes in Control and Information Sciences 185, R.F. Curtain, A. Bensoussan and J.L. Lions, eds., Springer Verlag, New York (1992) 347-365.

[25] A. Zeidler, Non linear functional analysis and its applications, Vol. 2, Springer Verlag, New York (1986). 